Revista Arbitrada Interdisciplinaria de Ciencias de la Salud. SALUD Y VIDA

Volumen 4. Número 7. Año 4. Enero - Junio 2020

Hecho el depósito de Ley: FA2016000010

ISSN: $2610-8038$

FUNDACIÓN KOINONIA (F.K)

Santa Ana de Coro, Venezuela.

Aida Monserrate Macias-Alvia; Tibisay Rincón-Ríos; Deysi Delgado-Lopez; Letty Soraida Quimi-Cobos

http://dx.doi.org/10.35381/s.v.v4i7.660

\title{
El Autocuidado como herramienta de atención primaria en estudiantes universitarios
}

\section{Self-care as a primary care tool in university students}

\author{
Aida Monserrate Macias-Alvia \\ aidita.macias@hotmail.com \\ Universidad Estatal del Sur de Manabí, Jipijapa \\ Ecuador \\ https://orcid.org/0000-0001-5290-4317 \\ Tibisay Rincón-Ríos \\ tibyrin@hotmail.com \\ Universidad del Zulia, Maracaibo \\ Venezuela \\ https://orcid.org/0000-0003-4288-1822 \\ Deysi Delgado-López \\ deysidelgadol@hotmail.com \\ Universidad Estatal del Sur de Manabí, Jipijapa \\ Ecuador \\ https://orcid.org/0000-0002-4930-3120 \\ Letty Soraida Quimi-Cobos \\ lettyquimic63@hotmail.com \\ Universidad Estatal del Sur de Manabí, Jipijapa \\ Ecuador \\ https://orcid.org/0000-0002-8577-5205
}

Recibido: 1 de octubre de 2019

Aprobado: 20 de diciembre de 2019

\section{RESUMEN}

El autocuidado como herramienta de atención primaria, necesita ser reforzado en la formación de los profesionales de Enfermería. Con base a esta premisa, se planteó en este artículo como objetivo evaluar el autocuidado como herramienta de atención primaria en estudiantes del quinto al octavo semestre de la carrera de enfermería de la Universidad Estatal del Sur de Manabí. Para ello, el estudio siguio una metodologia 
descriptiva y cuasi experimental. La población la constituyeron 187 estudiantes del quinto al octavo semestre de la carrera de enfermería de la universidad estatal del Sur de Manabí. La recolección de la información se realizó a través de un instrumento tipo cuestionario, y el análisis fue la estadística descriptiva y frecuencial. Entre sus conclusiones, se pudo pudo constatar que existe un estado regular de atención al autocuidado entre los estudiantes de la carrera de Enfermería a pesar de haber recibido conocimientos teóricos de esta herramienta.

Descriptores: Autocuidado; atención primaria de salud; hábitos; conductas y enfermería.

\section{SUMMARY}

Self-care as a primary care tool, needs to be strengthened in the training of Nursing professionals. Based on this premise, this article was raised to evaluate self-care as a primary care tool for students fifth through eighth semester of nursing career at Southern Manabí State University. To do this, the study followed a descriptive and quasi-experimental methodology. The population was made up of 187 students from the fifth to eighth semester of the nursing degree of the southern state university of South Manabí. The collection of the information was carried out through a questionnaire-like instrument, and the analysis was the descriptive and frequency statistics. Among her conclusions, it could be noted that there is a regular state of attention to self-care among students of the Nursing career despite having received theoretical knowledge of this tool.

Descriptors: Self-care; primary Health Care; habits; behaviors and nursing.

\section{INTRODUCCIÓN}

La enfermería, desde sus inicios, se ha considerado como un producto social vinculado al arte de cuidar, por lo que responde a la necesidad de ayudar a las personas, cuando estas no tienen capacidad suficiente para proporcionarse a sí misma o a las personas que dependen de ellas, la calidad y cantidad de cuidados para mantener la vida, identificando los problemas de salud y las necesidades reales y/o potenciales de la persona, familia y comunidad que demandan cuidados, generando autonomía o dependencia como consecuencia de las actividades que asuma el profesional de la enfermería (Bastías y Col, 2014). 
Cabe destacar, que los profesionales de la enfermería son un grupo social que cumple con dos tareas primordiales: el cuidado de los pacientes y la enseñanza de la enfermería; y ambas requieren de un compromiso moral con las necesidades de la sociedad. Diferentes autores reconocen que la enfermería es una ciencia humana práctica que usa las teorías de las ciencias naturales y de la conducta para comprender y desarrollar las prácticas del cuidado. El cuidar es esencia de la disciplina, siendo núcleo y por tanto objeto de estudio, debe ser discernido como tal, pero además reconocido por todo profesional de enfermería que decide serlo, y ejercerla con vocación, deber y responsabilidad humana (Medina Moya y col, 2006).

Por ello, el autocuidado en la actualidad, constituye un tema de trascendencia, porque mediante su conocimiento y aplicación permiten incrementar la proactividad de los trabajadores en el campo de la salud. La práctica del autocuidado requiere, en gran medida, cambio de actitud frente a los nuevos retos de la vida moderna. Las nuevas concepciones de salud y promoción de la salud proponen una nueva forma de asumir el autocuidado con rostro humano, como estrategia fundamental para promover la vida y el bienestar; por eso se requiere el desarrollo de habilidades personales para optar decisiones saludables, lo cual constituye uno de los cinco componentes de la promoción de la salud (Manturano Velásquez y col, 2013).

Siendo así, el autocuidado se constituye, entonces, en una herramienta esencial en la atención primaria en salud (APS) ya que tiene la finalidad de promover una vida saludable previniendo las enfermedades. Con preocupación hemos notado que el autocuidado está siendo poco fomentado, en los pacientes, por los profesionales de la Enfermería quienes se limitan a cumplir tratamientos indicados por el médico omitiendo las instrucciones adicionales que conllevan a que el paciente se preocupe por recuperar y mantener su salud y que son la base del autocuidado. Esta realidad puede tener su origen en la poca insistencia que se hace del autocuidado en los programas de formación de algunas carreras de la salud, donde debería existir, una especial 
insistencia en la relevancia de esta práctica la cual puede ser fomentada desde una propuesta didáctica aplicable durante toda la carrera. (Duarte E. y Col, 2013).

Este desinterés por el autocuidado lleva a los mismos estudiantes de Enfermería a sufrir enfermedades durante su carrera ya que no son capaces, en los casos donde sí hay una formación teórica sobre el autocuidado, de aplicarlo en ellos mismos, lo que los lleva a enfermarse de lo que ellos deberían evitar que padezcan los pacientes o la comunidad en general. Considerando la problemática anteriormente expuesta, se presenta en este artículo evaluar el autocuidado como herramienta de atención primaria en estudiantes del quinto al octavo semestre de la carrera de enfermería de la universidad estatal del Sur de Manabí.

\section{DESARROLLO}

El autocuidado, dentro de las clasificaciones para el estudio de las diferentes teorías y modelos, encontramos los modelos de suplencia o ayuda donde el rol fundamental de la enfermera consiste en suplir o ayudar a realizar acciones que la persona no puede llevar a cabo por si solas en un momento de su vida, acciones que preservan la vida, una de las representantes más importante de esta tendencia es la propuesta por Orem (1999), quien establece que estableciendo la teoría del déficit del autocuidado como un modelo general compuesto por tres subteorías relacionadas: La teoría de autocuidado, del déficit del autocuidado y la teoría de los sistemas de enfermería (Ministerio de Salud Pública. Código de Ética. Ecuador, 2013).

Autores como Benavent y Ferrer plantean que la teoría de Orem "Déficit de autocuidado" es una de la más estudiada y validada en la práctica de enfermería por la amplia visión de la asistencia de salud en los diferentes contextos que se desempeña este profesional, ya que logra estructurar los sistemas de enfermería en relación con las necesidades del autocuidado (Benavent y col, 2000.).

Orem (1999), definió los siguientes conceptos meta paradigmáticos: Persona: concibe al ser humano como un organismo biológico, racional y pensante. Como un todo 
integral dinámico con capacidad para conocerse, utilizar las ideas, las palabras y los símbolos para pensar, comunicar y guiar sus esfuerzos, capacidad de reflexionar sobre su propia experiencia y hechos colaterales a fin de llevar a cabo acciones de autocuidado dependiente. También, expone que para la ejecución de sus planteamientos, deben tenerse presente las siguientes pautas:

a. Examinar los factores, los problemas de salud y el déficit de autocuidado.

b. Realizar la recogida de datos acerca de los problemas y la valoración del conocimiento, habilidades motivación y orientación del cliente.

c. Analizar los datos para descubrir cualquier déficit de autocuidado, lo cual supondrá la base para el proceso de intervención de enfermería.

d. Diseñar y planificar la forma de capacitar y animar al cliente para que participe activamente en las decisiones del autocuidado de salud.

e. Poner el sistema de enfermería en acción y asumir un papel de cuidado u orientador, contando con la participación del cliente.

Para ello, la realización del autocuidado requiere de una acción intencionada y calculada, la que está condicionada por el conocimiento y el repertorio de habilidades de la persona y se basa en la premisa de que los individuos saben cuándo necesitan ayuda y, por lo tanto, son conscientes de las acciones específicas que necesitan realizar. Además, Lopez (2006), explica el autocuidado como aquellos actos que permiten definir los cuidados de enfermería con el fin de ayudar al individuo a llevar a cabo y mantener acciones de autocuidado para conservar la salud y la vida, recuperarse de la enfermedad y afrontar las consecuencias de esta.

En otro sentido, se destacan requisitos universales, son los que precisan todos los individuos para preservar y mantener su funcionamiento integral como persona e incluyen la conservación del aire, agua, alimentos, eliminación, la actividad y el descanso, soledad e interacción social, la prevención de riesgos y promoción de la actividad humana. Estos requisitos que exponemos a continuación representan los tipos 
Revista Arbitrada Interdisciplinaria de Ciencias de la Salud. SALUD Y VIDA

Volumen 4. Número 7. Año 4. Enero - Junio 2020

Hecho el depósito de Ley: FA2016000010

ISSN: $2610-8038$

FUNDACIÓN KOINONIA (F.K)

Santa Ana de Coro, Venezuela.

Aida Monserrate Macias-Alvia; Tibisay Rincón-Ríos; Deysi Delgado-Lopez; Letty Soraida Quimi-Cobos

de acciones humanas que proporcionan las condiciones internas y externas para mantener la estructura y la actividad, que a su vez apoyan el desarrollo y la maduración humana, fomentando positivamente la salud y el bienestar:

- Mantenimiento de un ingreso suficiente de aire.

- Mantenimiento de una ingesta suficiente de agua.

- Mantenimiento de una ingesta suficiente de alimentos

- Provisión de cuidados asociados con procesos de eliminación urinaria e intestinal.

- Equilibrio entre actividades y descanso.

- Equilibrio entre soledad y la comunicación social.

- Prevención de peligros para la vida, funcionamiento y bienestar humano.

- Promoción del funcionamiento humano, y el desarrollo dentro de los grupos sociales de acuerdo al potencial humano.

Cada uno de estos requisitos están íntimamente relacionados entre sí, pero cada uno de ellos son logrados en diferentes grados de acuerdo a los "factores condicionantes básicos" que son determinantes e influyentes en el estado de salud de toda persona. Los requisitos de cuidados personales del desarrollo, surgen como el resultado de los procesos de desarrollo o estados que afectan el desarrollo humano. Los de cuidados personales de los trastornos de salud, entre estos se encuentran los que se derivan de traumatismo, incapacidad, diagnóstico y tratamiento médico e implican la necesidad de introducir cambios en el estilo de vida. Con la aplicación de esta categoría el cuidado es intencionado, los inicia la propia persona este es eficaz y fomenta la independencia (Silva y col, 2006).

Teoría de déficit de autocuidado: Esta teoría es el núcleo del modelo de Orem (1999), establece que las personas están sujetas a las limitaciones relacionadas o derivadas de su salud, que los incapacitan para el autocuidado continuo, o hacen que el autocuidado 
Revista Arbitrada Interdisciplinaria de Ciencias de la Salud. SALUD Y VIDA

Volumen 4. Número 7. Año 4. Enero - Junio 2020

Hecho el depósito de Ley: FA2016000010

ISSN: $2610-8038$

FUNDACIÓN KOINONIA (F.K)

Santa Ana de Coro, Venezuela.

Aida Monserrate Macias-Alvia; Tibisay Rincón-Ríos; Deysi Delgado-Lopez; Letty Soraida Quimi-Cobos

sea ineficaz o incompleto. Existe un déficit de autocuidado cuando la demanda de acción es mayor que la capacidad de la persona para actuar, o sea, cuando la persona no tiene la capacidad y/o no desea emprender las acciones requeridas para cubrir las demandas de autocuidado. La existencia de un déficit de autocuidado es la condición que legitimiza la necesidad de cuidados de enfermería.

\section{Principios para la implementación del autocuidado en atención primaria de la salud}

El autocuidado posee unos principios que se deben tener en cuenta en su promoción:

a. Es un acto de vida que permite a las personas convertirse en sujetos de sus propias acciones. Por tanto, es un proceso voluntario de la persona para consigo misma.

b. Debe ser una filosofía de vida y una responsabilidad individual íntimamente ligada a la cotidianidad y a las experiencias vividas de las personas, pero a su vez debe estar fundamentado en un sistema de apoyo formal e informal como es el sistema social y el de salud.

c. Es una práctica social que implica cierto grado de conocimiento y elaboración de un saber y que da lugar a intercambios y relaciones interindividuales. (Taylor SG y col, 2007).

Promoción del autocuidado: Muñoz y col (2008), explican que las personas asumen el autocuidado como una práctica cotidiana de vida y salud, cuando incluyen en su promoción las siguientes estrategias:

a. Desarrollar en las personas autoestima y generar niveles de fortalecimiento o empoderamiento (empowerment), como estrategias que reviertan la internalización de la impotencia, favorezcan el sentido de control personal y desarrollen habilidades de movilización personal y colectiva para cambiar las condiciones personales y sociales en pro de la salud. 
b. Involucrar el diálogo de saberes, el cual permite identificar, interpretar y comprender la lógica y la dinámica del mundo de la vida de las personas mediante el descubrimiento y entendimiento de su racionalidad, sentido y significado, para poder articularlo con la lógica científica y recomponer una visión esclarecida de la enfermedad y de la salud que se traduzca en comportamientos saludables.

c. Explorar y comprender las rupturas que existen entre conocimiento, actitudes y prácticas, y configurar propuestas de acción y capacitación que hagan viable la armonía entre cognición y comportamientos.

d. Los agentes de salud deben asumir el autocuidado como una vivencia cotidiana, pues al vivir saludablemente, la promoción del autocuidado sería el compartir de sus propias vivencias. En la medida que el personal de salud viva con bienestar se estará en condiciones de promocionar la salud de los demás.

e. Contextualizar el autocuidado, es decir una direccionalidad de acuerdo con las características de género, etnia y ciclo vital humano. Es importante entender que cada persona tiene una historia de vida, con valores, creencias, aprendizajes y motivaciones diferentes.

f. Generar procesos participativos; la promoción del autocuidado debe incluir participación más activa e informada de las personas en el cuidado de su propia salu\}d, entendida la participación como el proceso mediante el cual la comunidad asume como propio el cuidado de sí misma y del ambiente que la rodea, dirigiendo la sensibilización a aumentar el grado de control y compromiso mutuo sobre su propia salud de las personas que ofrecen los servicios de salud y quienes lo reciben.

g. El agente de salud debe buscar espacios de reflexión y discusión acerca de lo que la gente sabe, vive y siente de las diferentes situaciones de la vida y la salud con lo cual identificar prácticas de autocuidado favorables, desfavorables e inocuas y promover, mediante un proceso educativo de reflexión-acción, un regreso a la práctica para transformarla. 
Revista Arbitrada Interdisciplinaria de Ciencias de la Salud. SALUD Y VIDA

Volumen 4. Número 7. Año 4. Enero - Junio 2020

Hecho el depósito de Ley: FA2016000010

ISSN: 2610-8038

FUNDACIÓN KOINONIA (F.K)

Santa Ana de Coro, Venezuela.

Aida Monserrate Macias-Alvia; Tibisay Rincón-Ríos; Deysi Delgado-Lopez; Letty Soraida Quimi-Cobos

\section{METODOLOGIA}

El estudio siguio una metodologia descriptiva y cuasi experimental, que según Hernández, Fernández y Baptista (2014), afirman que la investigación descriptiva es la que se orienta a redactar informes relacionados con el estado real de las personas, objeto, situaciones o fenómenos tal cual se presentan en el momento de su recolección, describe lo que mide sin analizar inferencias ni verificar hipótesis. La población la constituyeron 187 estudiantes del quinto al octavo semestre de la carrera de enfermería de la universidad estatal del Sur de Manabí. La recolección de la información se realizó a través de un instrumento tipo cuestionario, con escalas dicotómicas y de varias alternativas de repuestas. La validez del instrumento fue realizada a través del criterio de expertos en el área. La técnica de análisis fue la estadística descriptiva y frecuencial. Los criterios de inclusión refieren a estudiantes ubicados en los últimos dos años de la carrera a fin de asegurar que hayan cursado y aprobado las materias relacionadas con la atención primaria en salud, de la carrera de enfermería de la Universidad Estatal del Sur de Manabi, mayores de 18 años de edad, sin distingo de etnia, género o procedencia. Además, será necesaria para la inclusión en el estudio, la aceptación y firma del consentimiento informado.

\section{RESULTADOS}

De acuerdo a la metodología explicada a fin de cumplir con el objetivo propuesto en este artículo, se realizó el análisis y discusión de los resultados estadísticos de los datos aportados por los estudiantes investigados, en relación a los hábitos y conductas durante el autocuidado como herramienta de atención primaria en estudiantes del quinto al octavo semestre de la carrera de enfermería de la universidad estatal del sur de Manabí, los cuales se presentan en tablas con sus respectivos gráficos explicativos. 
Revista Arbitrada Interdisciplinaria de Ciencias de la Salud. SALUD Y VIDA

Volumen 4. Número 7. Año 4. Enero - Junio 2020

Hecho el depósito de Ley: FA2016000010

ISSN: 2610-8038

FUNDACIÓN KOINONIA (F.K)

Santa Ana de Coro, Venezuela.

Aida Monserrate Macias-Alvia; Tibisay Rincón-Ríos; Deysi Delgado-Lopez; Letty Soraida Quimi-Cobos

\section{Tabla №1}

Distribución frecuencial y porcentual del hábito tabáquico en estudiantes del quinto al octavo semestre de la carrera de enfermería de la universidad estatal del sur de Manabí.

\begin{tabular}{r|r|r|r|r}
\hline $\begin{array}{r}\text { Alternativas/ } \\
\text { Estadístico }\end{array}$ & FR & $\%$ & FRA & $\% A$ \\
\hline $\mathrm{SI}$ & 65 & 34,8 & 65 & 34,8 \\
\hline $\mathrm{NO}$ & 122 & 65,2 & 187 & 100 \\
\hline Total & 187 & 100,0 & 187 & 100 \\
\hline
\end{tabular}

Fuente: Elaboración propia (2020)

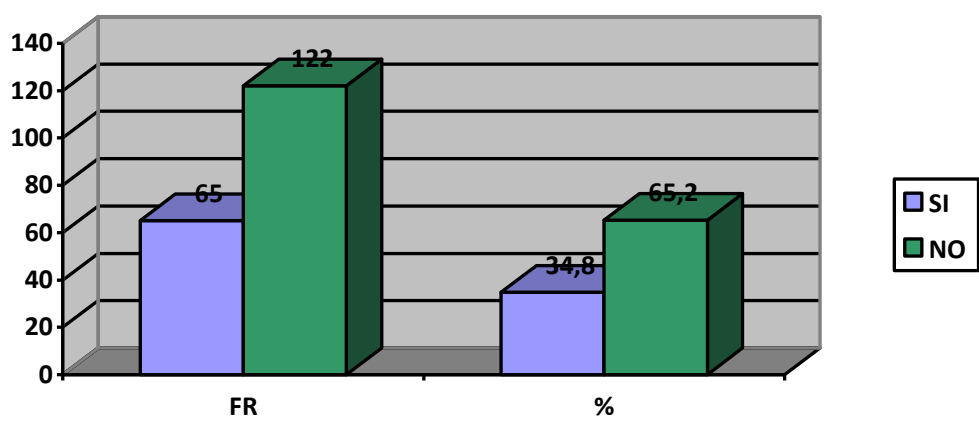

Gráfico №1. Distribución frecuencial y porcentual del hábito tabáquico en estudiantes del quinto al octavo semestre de la carrera de enfermería de la universidad estatal del sur de Manabí.

Fuente: Elaboración propia (2020)

En la tabla y grafico 1, se indica que el $65,2 \%$ de los estudiantes investigado coincidieron en opinar que no poseían el habito tabáquico. Seguido de la afirmación del resto de ellos, representado por el $34,8 \%$ del total. 
Revista Arbitrada Interdisciplinaria de Ciencias de la Salud. SALUD Y VIDA

Volumen 4. Número 7. Año 4. Enero - Junio 2020

Hecho el depósito de Ley: FA2016000010

ISSN: $2610-8038$

FUNDACIÓN KOINONIA (F.K)

Santa Ana de Coro, Venezuela.

Aida Monserrate Macias-Alvia; Tibisay Rincón-Ríos; Deysi Delgado-Lopez; Letty Soraida Quimi-Cobos

Tabla №2

Distribución frecuencial y porcentual del número de cigarrillos que consumen diariamente los estudiantes del quinto al octavo semestre de la carrera de enfermería de la universidad estatal del sur de Manabí.

\begin{tabular}{r|c|c|c|c}
\hline $\begin{array}{r}\text { Alternativas/ } \\
\text { Estadístico }\end{array}$ & FR & & FRA & \%A \\
\hline 1 a 3 & 12 & 6,4 & 12 & 6,4 \\
\hline 4 a 7 & 3 & 1,6 & 15 & 100 \\
\hline 8 a 11 & 0 & - & 15 & 100 \\
\hline Mas de 12 & & & & 15 \\
\hline Rara vez & 50 & 26,7 & 65 & 100 \\
\hline Nunca & 122 & 65,2 & 187 & 100 \\
\hline Total & 187 & 100 & 187 & 100 \\
\hline
\end{tabular}

Fuente: Elaboración propia (2020)
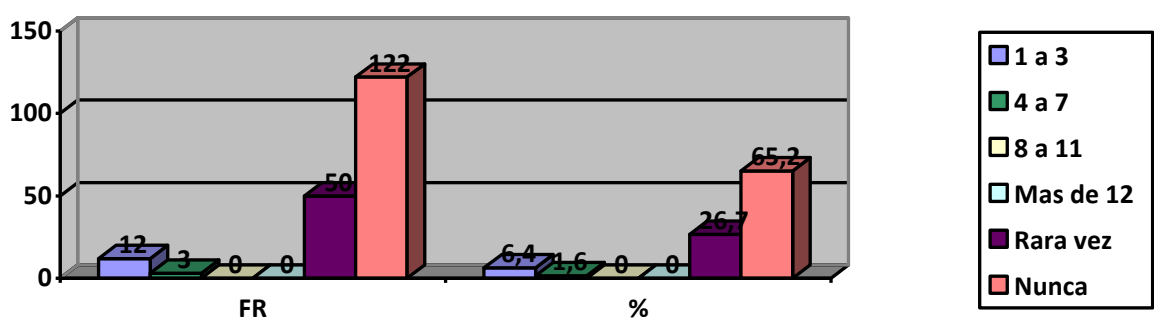

Gráfico №2. Distribución frecuencial y porcentual del número de cigarrillos que consumen diariamente los estudiantes del quinto al octavo semestre de la carrera de enfermería de la universidad estatal del sur de Manabí.

Fuente: Elaboración propia (2020) 
En la tabla y grafico 2, se evidenció, que el $65,2 \%$ de los estudiantes investigados coincidieron en opinar nunca habían consumido cigarrillos y rara vez lo hacen el $26,7 \%$ del total de los investigados. En menores porcentajes indicaron con el 6,4\% y 1,6\% quienes consumían entre 1 a3 cigarrillos y 4 a 7 cigarrillos respectivamente, Cabe destacar que entre 8 a 12 cigarrillos no se presentó opinión alguna.

Tabla №3

Distribución frecuencial y porcentual de la evasión de espacios con humo en estudiantes del quinto al octavo semestre de la carrera de enfermería de la universidad estatal del sur de Manabí.

\begin{tabular}{r|r|r|r|r}
\hline $\begin{array}{r}\text { Alternativas/ } \\
\text { Estadístico }\end{array}$ & FR & $\%$ & FRA & $\% A$ \\
\hline $\mathrm{SI}$ & 120 & 64,2 & 120 & 64,2 \\
\hline $\mathrm{NO}$ & 67 & 35,8 & 187 & 100 \\
\hline Total & 187 & 100,0 & 187 & 100 \\
\hline
\end{tabular}

Fuente: Elaboración propia (2020)

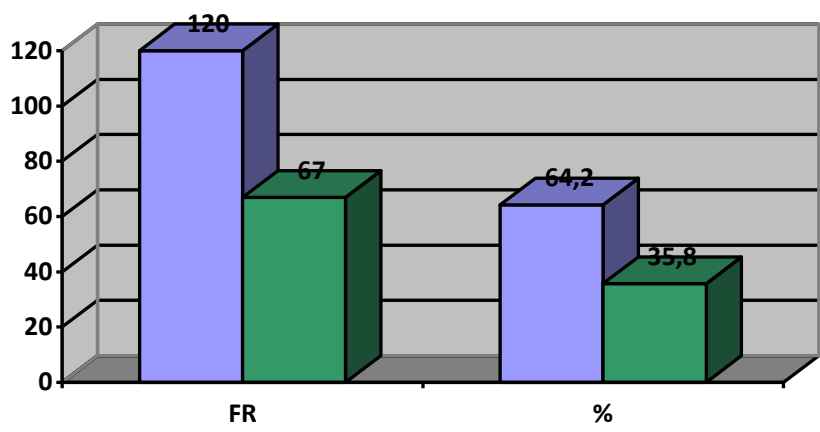

Gráfico №3. Distribución frecuencial y porcentual de la evasión de espacios con humo en estudiantes del quinto al octavo semestre de la carrera de enfermería de la universidad estatal del sur de Manabí.

Fuente: Elaboración propia (2020) 
En la tabla y grafico 3 , se indica que el $64,2 \%$ de los estudiantes investigado coincidieron en opinar que evadían estar en lugares o espacios con humo. Seguido de la negación del resto de ellos, representado por el 35,8\% del total.

\section{Tabla №4}

Distribución frecuencial y porcentual del consumo de bebidas alcohólicas en estudiantes del quinto al octavo semestre de la carrera de enfermería de la universidad estatal del sur de Manabí.

\begin{tabular}{r|r|r|r|r}
\hline $\begin{array}{r}\text { Alternativas/ } \\
\text { Estadístico }\end{array}$ & FR & $\%$ & FRA & $\% A$ \\
\hline $\mathrm{SI}$ & 84 & 44,9 & 84 & 44,9 \\
\hline $\mathrm{NO}$ & 103 & 55,1 & 187 & 100 \\
\hline Total & 187 & 100,0 & 187 & 100 \\
\hline
\end{tabular}

Fuente: Elaboración propia (2020)

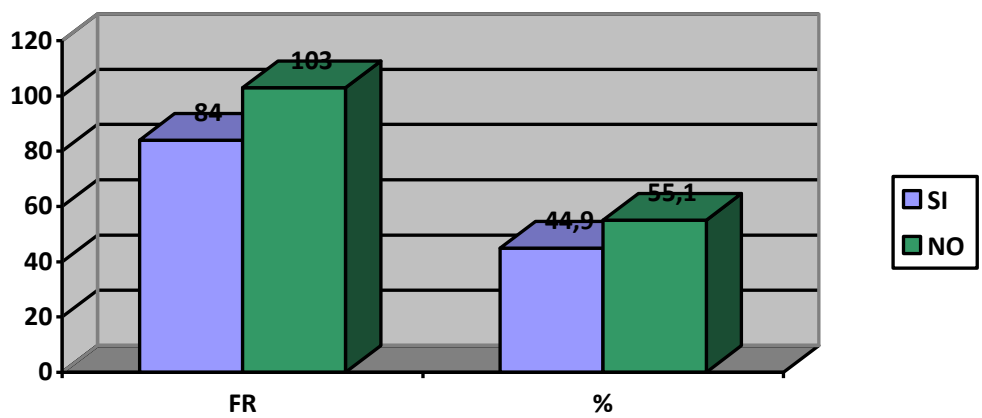

Gráfico №4. Distribución frecuencial y porcentual del consumo de bebidas alcohólicas en estudiantes del quinto al octavo semestre de la carrera de enfermería de la universidad estatal del sur de Manabí.

Fuente: Elaboración propia (2020) 
Revista Arbitrada Interdisciplinaria de Ciencias de la Salud. SALUD Y VIDA

Volumen 4. Número 7. Año 4. Enero - Junio 2020

Hecho el depósito de Ley: FA2016000010

ISSN: $2610-8038$

FUNDACIÓN KOINONIA (F.K)

Santa Ana de Coro, Venezuela.

Aida Monserrate Macias-Alvia; Tibisay Rincón-Ríos; Deysi Delgado-Lopez; Letty Soraida Quimi-Cobos

En la tabla y grafico 4 , se indica que el $55,1 \%$ de los estudiantes investigado coincidieron en opinar que no consumían bebidas alcohólicas. Seguido de la negación del resto de ellos, representado por el $44,9 \%$ del total.

Tabla №5

Distribución frecuencial y porcentual del estado de embriaguez en estudiantes del quinto al octavo semestre de la carrera de enfermería de la universidad estatal del sur de Manabí.

\begin{tabular}{r|r|r|r|r}
\hline $\begin{array}{r}\text { Alternativas/ } \\
\text { Estadístico }\end{array}$ & FR & $\%$ & FRA & $\% A$ \\
\hline $\mathrm{SI}$ & 84 & 44,9 & 84 & 44,9 \\
\hline $\mathrm{NO}$ & 103 & 55,1 & 187 & 100 \\
\hline Total & 187 & 100,0 & 187 & 100 \\
\hline
\end{tabular}

Fuente: Elaboración propia (2020)

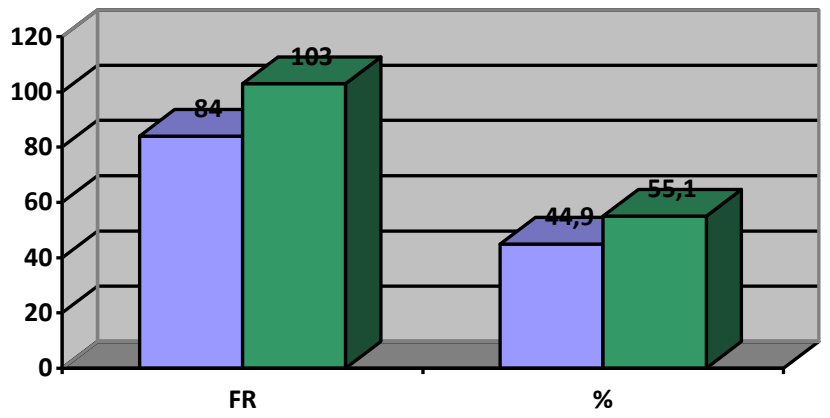

Gráfico №5. Distribución frecuencial y porcentual del estado de embriaguez en estudiantes del quinto al octavo semestre de la carrera de enfermería de la universidad estatal del sur de Manabí.

Fuente: Elaboración propia (2020) 
En la tabla y grafico 5, se indica que el $55,1 \%$ de los estudiantes investigado indicaron que nunca habían experimentado estado de embriaguez. Seguido de la afirmación del resto de ellos, representado por el $44,9 \%$ del total.

\section{Tabla №6}

Distribución frecuencial y porcentual del consumo de alcohol en estudiantes del quinto al octavo semestre de la carrera de enfermería de la universidad estatal del sur de Manabí.

\begin{tabular}{|c|c|c|c|c|}
\hline $\begin{array}{r}\text { Alternativas/ } \\
\text { Estadístico }\end{array}$ & FR & $\%$ & FRA & $\% A$ \\
\hline Diario & $\overline{0}$ & 0 & 0 & 0 \\
\hline Todas las semanas & 4 & 2,1 & 4 & 2,1 \\
\hline $\begin{array}{r}\text { Menos de } 4 \text { veces al } \\
\text { mes }\end{array}$ & 80 & 42,9 & 84 & 45 \\
\hline Ninguno & 103 & 55 & 187 & 100 \\
\hline Total & 187 & 100 & 187 & 100 \\
\hline
\end{tabular}

Fuente: Elaboración propia (2020)

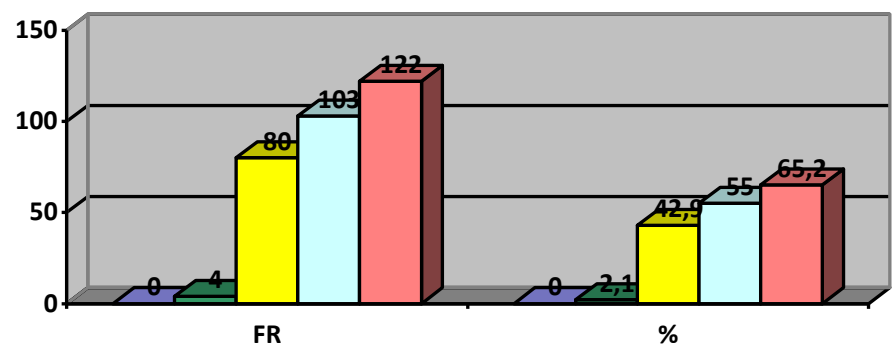

\begin{tabular}{|l|}
\hline Diario \\
$\square$ Todas las semanas \\
$\square$ Menos de 4 veces \\
$\square$ Ninguno \\
$\square$ Nunca \\
\hline
\end{tabular}

Gráfico №6. Distribución frecuencial y porcentual del consumo de alcohol en estudiantes del quinto al octavo semestre de la carrera de enfermería de la universidad estatal del sur de Manabí.

Fuente: Elaboración propia (2020) 
En la tabla y grafico 6 , se evidenció que el $55 \%$ de los estudiantes investigados coincidieron en opinar que nunca han consumido alcohol y el $42,9 \%$ menos de 4 veces al mes. En menores porcentajes indicaron con el 2,1\% que lo hacían toda la semana. Cabe destacar que de forma diaria ninguno presento opinión.

\section{Tabla №7}

Distribución frecuencial y porcentual del tipo de bebidas alcohólicas en estudiantes del quinto al octavo semestre de la carrera de enfermería de la universidad estatal del sur de Manabí.

\begin{tabular}{c|c|c|r|r}
\hline $\begin{array}{c}\text { Alternativas/ } \\
\text { Estadístico }\end{array}$ & FR & & FRA & \%A \\
\hline Cerveza & 53 & 28,3 & 53 & 28,3 \\
\hline Aguardiente & 2 & 1,1 & 55 & 29,4 \\
\hline Vodka & 5 & 2,7 & 60 & 32,1 \\
\hline Vino & 3 & 1,6 & 63 & 33,7 \\
\hline Whisky & 9 & 4,8 & 72 & 38,5 \\
\hline Ron & 1 & 0,5 & 73 & 39 \\
\hline Tequila & 2 & 1,1 & 75 & 40,1 \\
\hline Otros & 9 & 4,8 & 84 & 45.9 \\
\hline Ninguno & 103 & 55,1 & 187 & 100 \\
\hline
\end{tabular}

Fuente: Elaboración propia (2020)

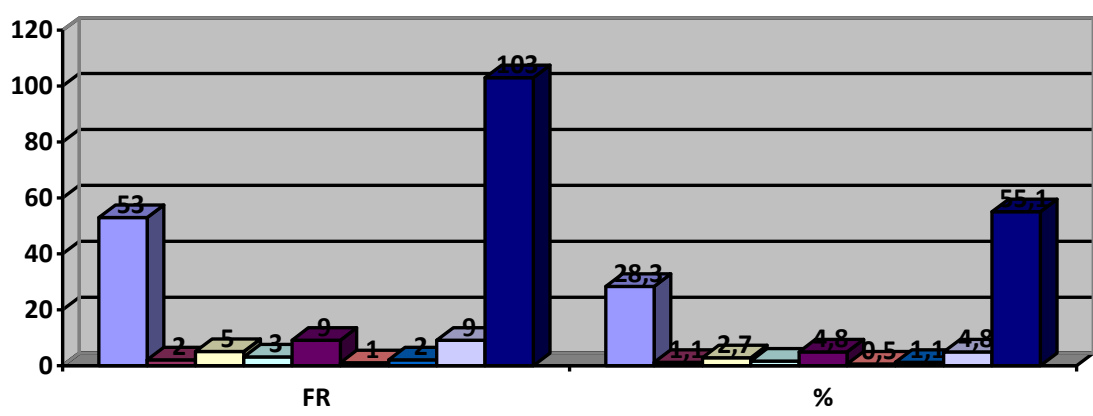

\begin{tabular}{|l}
$\square$ Cerveza \\
$\square$ Aguardiente \\
$\square$ Vodka \\
$\square$ Vino \\
$\square$ Whisky \\
$\square$ Ron \\
$\square$ Tequila \\
$\square$ Otros \\
$\square$ Ninguno
\end{tabular}

Gráfico №7. Distribución frecuencial y porcentual del tipo de bebidas alcohólicas en estudiantes del quinto al octavo semestre de la carrera de enfermería de la universidad estatal del sur de Manabí.

Fuente: Elaboración propia (2020) 
En la tabla y grafico 7 , se evidenció que el $55,1 \%$ de los estudiantes investigados coincidieron en opinar que nunca han consumido bebidas alcohólicas y el 28,3 indico que solo han consumido cerveza. "Por otra parte se indica que el 4,8\% había consumido Whisky y otras bebidas. En menor proporción con el 2,7 indico Vodka. Y solo $1,1 \%$ se inclinó por aguardiente y Tequila. Cabe destacra que el menor porcentaje de 0,5 señalo la alternativa Ron.

\section{Tabla №8}

Distribución frecuencial y porcentual del consumo de drogas ilícitas en estudiantes del quinto al octavo semestre de la carrera de enfermería de la universidad estatal del sur de Manabí.

\begin{tabular}{r|r|r|r|r}
\hline $\begin{array}{r}\text { Alternativas/ } \\
\text { Estadístico }\end{array}$ & FR & $\%$ & FRA & $\% A$ \\
\hline $\mathrm{SI}$ & 14 & 7,5 & 14 & 7,5 \\
\hline $\mathrm{NO}$ & 173 & 92,5 & 187 & 100 \\
\hline Total & 187 & 100,0 & 187 & 100 \\
\hline
\end{tabular}

Fuente: Elaboración propia (2020)

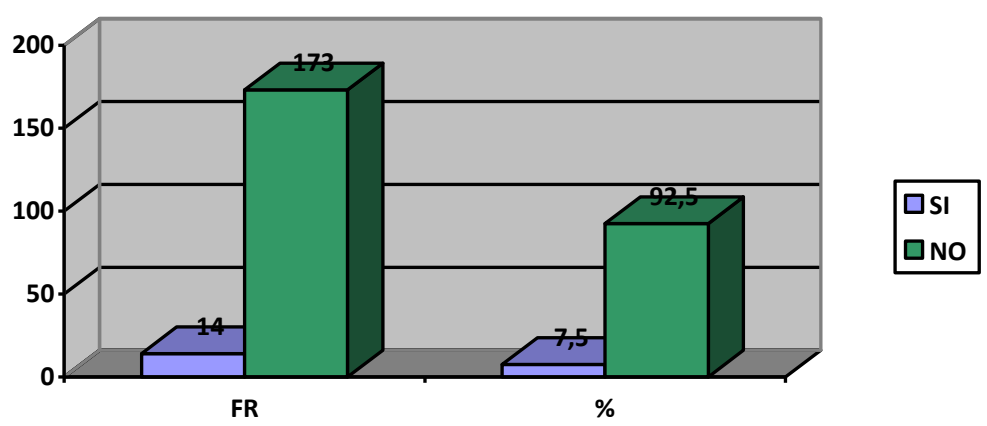

Gráfico №8. Distribución frecuencial y porcentual del consumo de drogas ilícitas en estudiantes del quinto al octavo semestre de la carrera de enfermería de la universidad estatal del sur de Manabí.

Fuente: Elaboración propia (2020) 
En la tabla y grafico 8 , se indica que el $92,5 \%$ de los estudiantes investigado coincidieron en opinar que no consumían drogas ilícitas. Seguido de la afirmación del resto de ellos, representado por el $7,5 \%$ del total.

\section{Tabla №9}

Distribución frecuencial y porcentual del consumo de actividades físicas semanales en estudiantes del quinto al octavo semestre de la carrera de enfermería de la universidad estatal del sur de Manabí.

\begin{tabular}{r|r|r|r|r}
\hline $\begin{array}{r}\text { Alternativas/ } \\
\text { Estadístico }\end{array}$ & FR & $\%$ & FRA & $\% A$ \\
\hline SI & 50 & 26,7 & 50 & \\
\hline NO & 137 & 73,3 & 187 & 100 \\
\hline Total & 187 & 100,0 & 187 & 100 \\
\hline
\end{tabular}

Fuente: Elaboración propia (2020)

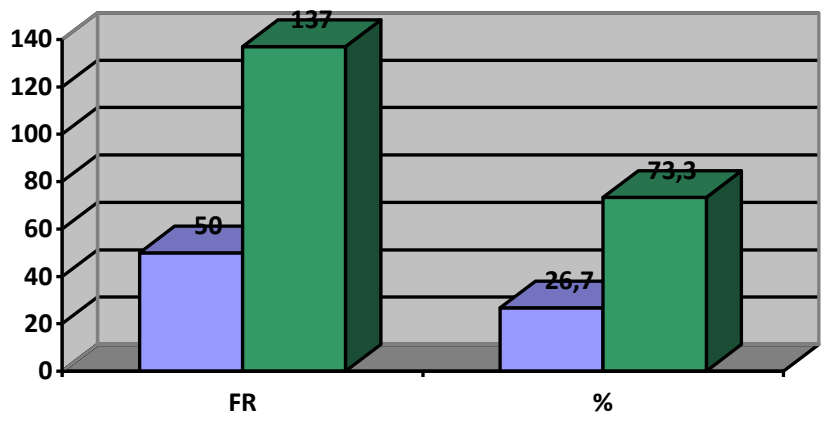

Gráfico №9. Distribución frecuencial y porcentual del consumo de actividades físicas semanales en estudiantes del quinto al octavo semestre de la carrera de enfermería de la universidad estatal del sur de Manabí.

Fuente: Elaboración propia (2020) 
En la tabla y grafico 9 , se indica que el $73,3 \%$ de los estudiantes investigado coincidieron en opinar que no realizaban actividades físicas semanales. Seguido de la afirmación del resto de ellos, representado por el $26,7 \%$ del total.

Tabla №10

Distribución frecuencial y porcentual del número de horas que duermen por la noche en estudiantes del quinto al octavo semestre de la carrera de enfermería de la universidad estatal del sur de Manabí.

\begin{tabular}{r|r|r|r|r}
\hline $\begin{array}{r}\text { Alternativas/ } \\
\text { Estadístico }\end{array}$ & FR & $\%$ & FRA & $\% A$ \\
\hline Más de 8 hrs & 39 & 20,9 & 39 & 20,9 \\
\hline Entre 5 y 7 hrs & 112 & 59,9 & 151 & 80,8 \\
\hline Entre 3 y 4 hrs & 29 & 15,5 & 180 & 96,3 \\
\hline Menos de 2 hrs & 7 & 3,7 & 187 & 100 \\
\hline
\end{tabular}

Fuente: Elaboración propia (2020)

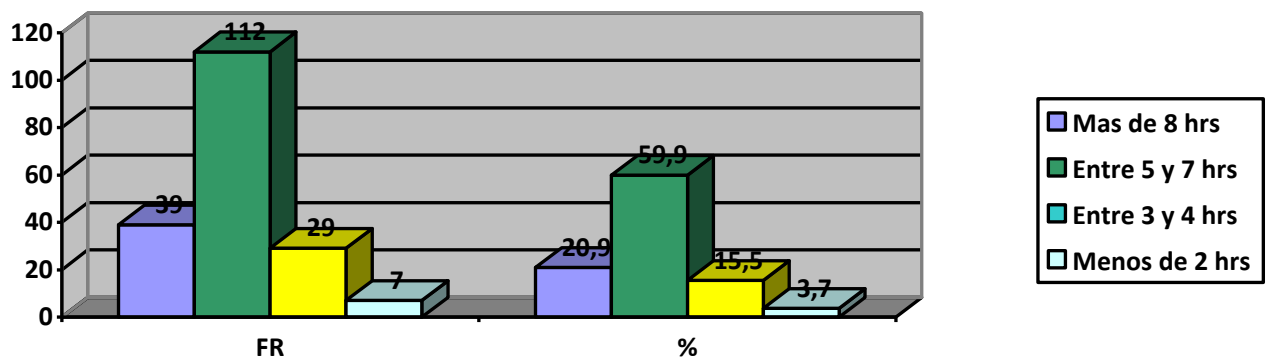

Gráfico №10. Distribución frecuencial y porcentual del número de horas que duermen por la noche en estudiantes del quinto al octavo semestre de la carrera de enfermería de la universidad estatal del sur de Manabí.

Fuente: Elaboración propia (2020) 
Revista Arbitrada Interdisciplinaria de Ciencias de la Salud. SALUD Y VIDA

Volumen 4. Número 7. Año 4. Enero - Junio 2020

Hecho el depósito de Ley: FA2016000010

ISSN: $2610-8038$

FUNDACIÓN KOINONIA (F.K).

Santa Ana de Coro, Venezuela.

Aida Monserrate Macias-Alvia; Tibisay Rincón-Ríos; Deysi Delgado-Lopez; Letty Soraida Quimi-Cobos

En la tabla y grafico 10 , se evidenció que el $59,9 \%$ de los estudiantes investigados coincidieron en opinar que duermen más de 8 horas diarias y el 20,9\% indico que dormían entre 5 a 7 horas diarias. "Por otra parte se indica que el 15,5\% solían dormir entre 3 y 4 horas. En menor proporción señalo con solo el 3,7\% que dormían menos de dos horas.

\section{CONCLUSIONES}

Dentro de las conclusiones una vez obtenidos los resultados se pueden mencionar los siguientes:

Se pudo constatar un estado regular de atención al autocuidado, entre los estudiantes de la carrera de Enfermería a pesar de haber recibido conocimientos teóricos de esta herramienta, previsto al obtener parcialmente las aceptaciones y afirmaciones de aquellos hábitos y conductas que son de atención primaria.

Existe una negación de más de la mitad de los estudiantes investigados en tener algún habito tabáquico, ni en haber consumido algún cigarrillos al igual en evadir lugares o espacios con humo. También, negaron el consumo de drogas ilícitas.

Se constató que los estudiantes de la carrera de enfermería no realizan actividades físicas semanales y duermen más de 8 horas diarias.

Predomino en la opinión por parte de los estudiantes investigados, la negación de haber experimentado en algún momento estado de embriagues y consumido bebidas alcohólicas.

\section{REFERENCIAS CONSULTADAS}

1. Bastías, E. y Stiepovich, B. (2014). Una revisión de los estilos de vida de estudiantes universitarios Iberoamericanos. Ciencia y enfermería, 20(2): 93-101.

2. Benavent MA, Ferrer F.(2000). Fundamentos de enfermería. Madrid: DAE Grupo Paradigmas. Enfermería 21. 


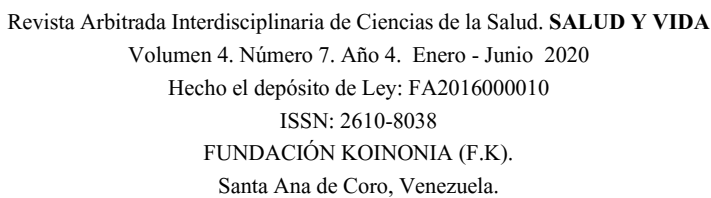

Aida Monserrate Macias-Alvia; Tibisay Rincón-Ríos; Deysi Delgado-Lopez; Letty Soraida Quimi-Cobos

3. Hernández, H. Fernández, C. y Baptista, P. (2010). Metodología de la Investigación. México: Mc Graw Hill Interamericana.

4. López, A. y Guerrero, S. (2006). Perspectiva internacional del uso de la teoría general de Orem. Investigación y Educación en Enfermería. 24(2), 90-100.

5. Manturano Velásquez Z, Cabanillas Chávez MT. (2014). Conocimiento y práctica de autocuidado del profesional de Enfermería del servicio de Pediatría del Hospital Nacional Guillermo Almenara Irigoyen. 2013. Revista Científica de Ciencias de la Salud. 7 (2): 35-42.

6. Medina Moya JL, Castillo Parra S. (2006). La enseñanza de la enfermería como una práctica reflexiva. Texto Contexto Enferm, Florianópolis; 15(2):303-311.

7. Ministerio de Salud Pública. Código de Ética. Ecuador. (2013). rhttp://instituciones.msp.gob.ec/somossalud/images/documentos/guia/Doc_Codig o_Etica.pdf

8. Muñoz M. y Cabieses B. (2008). Universidades y promoción de la salud: ¿cómo alcanzar el punto de encuentro? Revista Panam Salud Pública. Vol. 24(2):139146).

9. Orem, D. Modelos y teorías de enfermería. Barcelona: Harcourt Brace. Orem, D. E. (1999). Conceptos de enfermería en la práctica, México: Ediciones Científicas y Técnicas, S.A.

10.Taylor SG y Dorothea E. Orem. (2007). Teoría del déficit de autocuidado. En: Marriner A, Raile M. Modelos y teoría en enfermería. 6aㅡ ed. España: Elsevier; $p$. 267-295.

11.Silva IJ, Oliveira MFV, Silva SED, Polaro SHI, Radünz V, Do Santos EKA. (2009). Cuidado, autocuidado e cuidado de si: uma compreensão paradigmática para o cuidado de enfermagem. Rev Esc Enferm USP. 2009; 43 (3):697-703.

(C2020 por los autores. Este artículo es de acceso abierto y distribuido según los términos y condiciones de la licencia Creative Commons Atribución-NoComercial-Compartirlgual 4.0 Internacional (CC BY-NC-SA 4.0)

(https://creativecommons.org/licenses/by-nc-sa/4.0/). 